\begin{tabular}{|c|c|c|c|}
\hline$*$ & $\alpha 1906.0$ & $\delta 1906.0$ & Autorität \\
\hline 7 & $11^{\mathrm{h}} 20^{\mathrm{m}} 59^{\mathrm{s}} \cdot 44$ & $+2^{\circ} 23^{\prime} 23^{\prime \prime} .5$ & AG. Albany 4271 \\
\hline 8 & I I $20 \quad 43.43$ & +22858.5 & $\begin{array}{c}\text { BD. }+2^{\circ} 24239^{\mathrm{m}} 3 \text {, Anschl. } \\
\text { an } * 7 \text { (Ep. 1906.29) }\end{array}$ \\
\hline 9 & I I $19 \quad 55.47$ & $+23^{6} 45.9$ & AG. Albany 4265 \\
\hline Io & I1 1936.16 & +2264.9 & $\gg \quad \gg \quad 4264$ \\
\hline I I & II 2126.53 & +2 I 326.9 & $\begin{array}{l}\text { BD. }+2^{\circ} 24279^{\mathrm{m}} \cdot 5 \text {, Anschl. } \\
\text { an } * 12 \text { (Ep. } 1906.35)\end{array}$ \\
\hline 12 & I I $20 \quad 59.44$ & +22323.5 & AG. Albany 427 I \\
\hline I 3 & I I 2211.76 & +2 I 31.7 & $\begin{array}{l}\text { BD. }+2^{\circ} 24299^{m_{5}, \text { Anschl. }} \\
\text { an } * \text { I } 4 \text { (Ep. I } 906.36)\end{array}$ \\
\hline
\end{tabular}

\begin{tabular}{|c|c|c|c|}
\hline * & $\alpha 1906.0$ & $\delta 1906.0$ & Autorität \\
\hline I 4 & I I $22^{\mathrm{m}} 3^{2} \stackrel{\mathrm{s}}{9} 99$ & $+I^{\circ} 53^{\prime} 3^{6.8} .8$ & Áं. Albany 4280 inkl. EB. \\
\hline I 5 & I I $23 \quad 4.49$ & +15927.3 & $\gg \quad \gg \quad 4283$ \\
\hline I 6 & I I $23 \quad 58.01$ & $+143 \times 7.3$ & $\gg \quad>4290$ \\
\hline I 7 & I I $35 \quad 7.23$ & to 3144.4 & $\begin{array}{l}\text { I } 2^{\mathrm{m}}, \text { Anschluß an } * \text { I } 8 \\
(\text { Ep. 1906.44) }\end{array}$ \\
\hline ז 8 & I $35 \quad 30.69$ & +o 3152.0 & $\begin{array}{l}\text { BD. }+0^{\circ} 28 \times 899^{m}, 5, \text { Anschl. } \\
\text { an } * \text { I } 9 \text { (Ep. 1 } 906.45)\end{array}$ \\
\hline I9 & I I $34 \quad 34.55$ & to 28 I 9.5 & $\begin{array}{l}1 / 2\left(\text { Gött }_{1} 2921+\mathrm{BB} . \mathrm{VI}\right. \\
\left.+0^{\circ} \mathrm{o}_{21} 6\right)\end{array}$ \\
\hline
\end{tabular}

Bemerkungen.

Das physische Aussehen des Kometen unterlag während der Dauer dieser Beobachtungen nur geringfügigen Änderungen, deren Konstatierung überdies bei der Schwäche des Objekts mit Schwierigkeiten verknüpft war. In der ersten Zeit ließ sich in dem kleinen rundlichen Nebel ein scharfer Kern wahrnehmen, der bis März 17 im Zentrum des gleichförmig blassen Nebels aufleuchtete. Am 17. und 27. März schien der Kern exzentrisch zum nachfolgenden Ende hin gedrängt, wie wenn in der vorangehenden Richtung ein Schweif sich bilde. Am 28. März erblickte ich einen kurzen

\begin{tabular}{|c|c|c|c|c|c|c|}
\hline I906 & $\begin{array}{c}\text { Beob. } \\
\text { Kern- } \\
\text { helligkeit }\end{array}$ & $\begin{array}{c}\text { Beob. } \\
\text { Total- } \\
\text { helligkeit }\end{array}$ & $\begin{array}{c}\text { Reduktion } \\
\text { auf } \\
\text { März } 3\end{array}$ & $\begin{array}{c}\text { Total- } \\
\text { helligkeit } \\
\text { für März } 3\end{array}$ & $\begin{array}{l}\text { Schweif- } \\
\text { richtung }\end{array}$ & $\begin{array}{l}\text { Durch- } \\
\text { messer }\end{array}$ \\
\hline März $5^{1}$ ) & I $4^{\mathrm{m}}:$ & I I $\stackrel{\mathrm{m}}{\circ}$ & $0^{m} \cdot 0$ & I I $\stackrel{m_{0}}{\circ}$ & - & $20^{\prime \prime}$ \\
\hline $\left.7^{1}\right)$ & - & I 1.5 & 0.0 & I 1.5 & - & - \\
\hline 17 & I I. 5 & I 1.0 & -0.1 & 10.9 & $270^{\circ}$ & o: 7 \\
\hline 2 I & I 1.5 & I I.O & -0.1 & 10.9 & - & I. 5 \\
\hline 26 & I I. 5 & 11.0 & -0.2 & 10.8 & - & - \\
\hline 27 & I 1.5 & I I .O & -0.2 & 10.8 & 240 & 1,2 \\
\hline 28 & I I. 5 & I 1.0 & -0.2 & 10.8 & 250 & 0.9 \\
\hline April $\quad 2^{1}$ ) & 12 & I I . 5 & -0.2 & I I. 3 & - & 1: \\
\hline$\left.\quad 3^{1}\right)$ & I 2.5 & 12.0 & -0.2 & I 1.8 & - & $20^{\prime \prime}$ \\
\hline $\left..9^{1}\right)$ & - & I 2.5 & -0.2 & I 2.3 & - & IO \\
\hline
\end{tabular}

făcherartigen Schweif von I $20^{\circ}$ Öffnungswinkel, und diese Schweiferscheinung glaube ich noch bis Mai 14 zu erkennen. Später ist das Gebilde nur mehr rundlich mit zentralem Kern, und am letzten Beobachtungstage, Juni I 2, hat der Kern einer mäßig ausgeprägten Verdichtung Platz gemacht.

Über die bei 207 -facher Vergrößerung am $49 \mathrm{~cm}$ Refraktor erhaltenen Schätzungen der Helligkeit, der Schweifrichtung und des Durchmessers des Kometen gibt die Tabelle Auskunft. Der Reduktion der Helligkeit auf März 3 liegt das $I: r^{2} \Delta^{2}$-Gesetz zugrunde.

\begin{tabular}{|c|c|c|c|c|c|c|c|}
\hline \multicolumn{2}{|c|}{ I906 } & $\begin{array}{c}\text { Beob. } \\
\text { Kern- } \\
\text { helligkeit }\end{array}$ & $\begin{array}{c}\text { Beob. } \\
\text { Total- } \\
\text { helligkeit }\end{array}$ & \begin{tabular}{|c} 
Reduktion \\
auf \\
März 3
\end{tabular} & $\begin{array}{c}\text { Total- } \\
\text { helligkeit } \\
\text { für Mürz } 3\end{array}$ & $\begin{array}{l}\text { Schweif- } \\
\text { richtung }\end{array}$ & $\begin{array}{l}\text { Durch- } \\
\text { messer }\end{array}$ \\
\hline \multicolumn{2}{|c|}{ April I 2} & I $2 \stackrel{m}{\cdot} 5$ & $12 \mathrm{~m}$ & $-0^{m} \cdot 3$ & I I $\stackrel{m}{\cdot} 7$ & - & $20^{\prime \prime}$ \\
\hline & 17 & 12.5 & 11.5 & -0.3 & II.2 & $250^{\circ}$ & $0: 6$ \\
\hline & 21 & 12.5 & 12.0 & -0.4 & I 1.6 & 270 & 0.5 \\
\hline & 24 & 13 & I 2.0 & -0.4 & I 1.6 & 270 & 0.6 \\
\hline & 25 & I 3 & 12.5 & -0.4 & 12.1 & 300: & 0.8 \\
\hline \multirow[t]{5}{*}{ Mai } & IO & 13.5 & 12.5 & -0.6 & 11.9 & 270 & 0.4 \\
\hline & I 4 & 13.5 & 13.0 & -0.7 & I 2.3 & 270 & $15^{\prime \prime}$ \\
\hline & I 6 & I $3.5:$ & I 3.0 & -0.7 & 12.3 & - & I 5 \\
\hline & 22 & I 3.5 & I 3.0 & -0.8 & 12.2 & - & I 5 \\
\hline & 23 & I 3 & I 2.5 & -0.8 & I 1.7 & - & 30 \\
\hline Juni & 12 & - & I 3.5 & -1.1 & I 2.4 & - & 25 \\
\hline
\end{tabular}

Die schweifartige Ausströmung war also nach Ausweis des PW. ungefähr der Sonne zugewandt. *) - Juni 15 , 20, 2 I konnte der Komet nicht mehr aufgefunden werden; er verschwindet am dämmerhellen Abendhimmel.

Straßburg, 1906 Juni.

C. W. Wirtz.

*) Es ist dies offenbar dasselbe Phänomen, welches E. E. Barnard im 40-Zöller der Yerkessternwarte als zweiten Kern wahrgenommen hat; vgl. A. J. $586 . \quad K r$.

Osservazioni della cometa 1905 VI (1906 a).

Equatoriale di Fraunhofer $(0.175 \mathrm{~m})$. Micrometro anulare.

\begin{tabular}{|c|c|c|c|c|c|c|c|c|c|c|c|c|}
\hline 1906 & T.m. Napoli & $\Delta \alpha$ & & $\Delta \delta$ & Oss. & \multicolumn{2}{|c|}{$\alpha$ app. } & $\log p . \Delta$ & \multicolumn{2}{|c|}{$\delta$ app. } & $\log p \cdot \Delta$ & $*$ \\
\hline .24 & $\operatorname{II}^{\mathrm{h}} 44^{\mathrm{m}} 54^{\mathrm{s}}$ & $+\mathrm{I}^{\mathrm{m}} 50^{\mathrm{s}} \cdot 4$ & 一 & & $\mathbf{N}$ & $6^{\mathrm{h}} \times 7^{\mathrm{l}}$ & & 0.147 & $+74^{\circ} 4^{\circ}$ & & & \\
\hline 24 & I $\quad 56 \quad 17$ & $+I 48.72$ & - & 86.2 & G & $6 \mathrm{I} 7$ & $27 \cdot 74$ & 0.156 & +7439 & 26.5 & $0.263 n$ & \\
\hline 24 & 12427 & +148.02 & - & $8 \quad 26.5$ & $\mathbf{N}$ & 617 & 27.04 & 0.165 & $\begin{array}{r}7439 \\
\end{array}$ & 6.2 & $0.213 n$ & \\
\hline 24 & 12 I 33 & +141.08 & - & $9 \quad 14.3$ & G & 617 & 20.10 & 0.173 & $+743^{8}$ & 18.4 & $0.155 n$ & \\
\hline 25 & 94522 & +354.66 & + & 944.0 & $\mathbf{N}$ & 610 & 38.24 & 9.864 & +738 & 54.8 & $0.596_{n}$ & \\
\hline 25 & $\begin{array}{lll}9 & 57 & 43\end{array}$ & $\begin{array}{r}+349.46 \\
\end{array}$ & + & 851.1 & G & 610 & 33.04 & 9.904 & +73 & 1.9 & $0.576_{n}$ & \\
\hline
\end{tabular}




\begin{tabular}{|c|c|c|c|c|c|c|c|c|c|c|}
\hline 1906 & T.m. Napoli & $\Delta \alpha$ & $\Delta \delta$ & Oss. & $\alpha$ app. & $\log p \cdot \Delta$ & $\delta$ a & pp. & $\log p .4$ & * \\
\hline Febb.25 & $10^{\mathrm{h}} \quad 7^{\mathrm{m}} 40^{\mathrm{s}}$ & $+3^{m} 46: 88$ & $+7^{\prime} 56: 3$ & $\mathbf{N}$ & $6^{\mathrm{h}} 10^{\mathrm{m}} 30^{\mathrm{s}} \cdot 4^{6}$ & 9.933 & $+73^{\circ}$ & $7^{\prime} \quad 7^{\prime \prime} \mathrm{I}$ & $0.55^{8} n$ & 2 \\
\hline 25 & 1019 I7 & +344.94 & +733.8 & G & 6 10 28.52 & 9.964 & +73 & 644.6 & $0.535 n$ & 2 \\
\hline 27 & I I $\quad 8 \quad 32$ & $-5 \quad 14.41$ & $-10 \quad 24.4$ & $\mathbf{N}$ & $\begin{array}{lll}5 & 59 & 43.76\end{array}$ & 0.018 & +695 & $50 \quad 20.4$ & $0.064 n$ & 3 \\
\hline 27 & $\begin{array}{llll}\text { I I } & 17 & \text { IO }\end{array}$ & $-5 \times 5.54$ & $-10 \quad 55.6$ & G & $\begin{array}{llll}5 & 59 & 42.63\end{array}$ & 0.029 & +694 & 4949.2 & $0.078_{n}$ & 3 \\
\hline 27 & $\begin{array}{lll}11 & 25 & 47\end{array}$ & $-5 \times 6.67$ & -1137.6 & $\mathbf{N}$ & 55941.50 & 0.040 & +694 & 497.2 & $0.116_{n}$ & 3 \\
\hline Marz. 4 & $1442 \quad 21$ & -546.73 & +520.8 & G & $\begin{array}{lll}5 & 46 & 33.78\end{array}$ & $9.93^{8}$ & +622 & 240.1 & 0.806 & 4 \\
\hline 4 & $14 \quad 53 \quad 54$ & $-5 \quad 47.59$ & +23.4 & G & $5 \quad 46 \quad 32.92$ & 9.926 & +622 & 2042.7 & 0.815 & 4 \\
\hline 5 & I 5 & +I 34.15 & +426.5 & G & $\begin{array}{lll}5 & 45 & 6.02\end{array}$ & 9.644 & +605 & $58 \quad 53.8$ & 0.894 & 5 \\
\hline 5 & I 46 & +131.09 & +342.6 & $\mathbf{N}$ & 545 & $9.64 \mathrm{I}$ & +605 & $9 \cdot 9$ & 0.896 & 5 \\
\hline
\end{tabular}

Posizioni medie delle stelle di confronto.

\begin{tabular}{|c|c|c|c|}
\hline * & $\alpha 1906.0$ & $\delta$ I 906.0 & Autorità \\
\hline I & $6^{\mathrm{h}}+5^{\mathrm{m}} 37^{\mathrm{s}} \circ 9$ & $+74^{\circ} 47^{\prime} 25^{\prime \prime} 4$ & Kam. I ro95 \\
\hline 2 & $\begin{array}{lll}6 & 6 & 42.01\end{array}$ & $+7259 \quad 3.4$ & Gro. 1082 \\
\hline 3 & $\begin{array}{lll}6 & 4 & 56.97\end{array}$ & $+70 \circ 37.8$ & AG. Christ. 996 \\
\hline
\end{tabular}

\begin{tabular}{|c|c|c|c|}
\hline$*$ & a. I 906.0 & $\delta$ I 906.0 & Autorità \\
\hline 4 & $5^{\mathrm{h}} 52^{\mathrm{m}} 19^{\mathrm{g}} \cdot 95$ & $+62^{\circ}$ 1 $8^{\prime} 33^{\prime \prime} 5$ & AG. Hels. 4279 \\
\hline 5 & $\begin{array}{lll}5 & 43 & 3 \\
\mathrm{I} & 44\end{array}$ & +605421.6 & $\gg \quad \gg \quad 4206$ \\
\hline
\end{tabular}

Annotazioni.

Febbr. 27. Osservazione difficilissima per la estrema tenuita della immagine della cometa che apparisce senza alcuna forma definita e come un incerto bagliore nel campo del cannocchiale. - Marzo 4. Osservazioni fatte dopo i] tramonto della Luna in condizioni sfavorevoli per la presenza

di densi vapori. - Marzo 5. Le osservazioni sono state sospese perchè ostacolate dalla luce del crepuscolo. Ciascuna delle due osservazioni risulta come media da due altre eseguite dal medesimo osservatore.

R. Osservatorio astronomico di Capodimonte, r9o6 Giugno 18.

Vittorio Nobile, Eugenio Guerrieri.

\section{Mnemonic Rule for Gauss's trigonometrical formulae.}

In the Astr. Nachr. No. 4109 Herr Robert Vogel proposes a mnemonic for enabling one to write down Gauss's or as I prefer to call them Delambre's, analogies in spherical trigonometry. I suppose that everyone who has occasion frequently to employ these expressions makes use of some device or other to aid his memory. The following easily remembered rule has been used by tne for some time past and has proved most satisfactory. It may be worth while to place it on record.

Each of these analogies expresses the equality of two terms, one of which is of the form

$$
\text { A }\left(\begin{array}{c}
\text { sum } \\
\text { difference }
\end{array}\right) \text { in one set is always associated with a }\left(\begin{array}{c}
\text { cosine } \\
\text { sine }
\end{array}\right) \text { in the other. }
$$

This simple rule enables us at once to write down the four equations. Thus, suppose for example that we require the analogy containing $\sin 1 / 2(B+C)$. Since it is the sum we are given we can at once write down

$$
\sin 1 / 2(B+C) \cdot \cos 1 / 2 a=\cos 1 / 2() \cdots
$$

And since it is the sine we are given we see that the quantity inside the brackets is $b-c$, and therefore

$\sin 1 / 2(B+C) \cdot \cos 1 / 2 a=\cos 1 / 2(b-c) \cdots$

Since now we have the difference of $b$ and $c$ we know that it must be associated with $\sin 1 / 2 A^{\prime}$, and therefore

Radcliffe Observatory, Oxford, Igo6 October 9.

$$
\sin 1 / 2(B \pm C) \cdot \sin 1 / 2 a
$$

and the other of the form,

$$
\sin 1 / 2(b \pm c) \cdot \frac{\sin 1 / 2 A^{\prime}}{\cos } \quad\left(\text { where } A^{\prime}=\pi-A\right) .
$$

In these there are two sets of terms involved, namely,

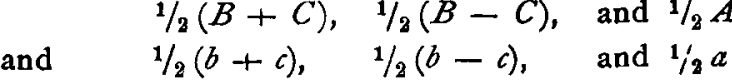

and the rule may be thus stated:

$$
\begin{aligned}
\sin 1 / 2(B+C) \cdot \cos 1 / 2 a & =\cos 1 / 2(b-c) \cdot \sin 1 / 2 A^{\prime} \\
\text { or } \quad \sin 1 / 2(B+C) \cdot \cos 1 / 2 a & =\cos 1 / 2(b-c) \cdot \cos 1 / 2 A .
\end{aligned}
$$

These four steps may, of course, all be taken at once and the formula written down in one line.

The necessity of introducing the quantity $A^{\prime}$ might have been avoided by taking for $A, B$, and $C$ the sides of the supplemental triangle, but a want of symmetry in the signs of the expressions would thus be introduced, and the rule as given above seems more convenient in practice. 